\title{
Value of multiphase contrast-enhanced CT with three-dimensional reconstruction in detecting depth of infiltration, lymph node metastasis, and extramural vascular invasion of gastric cancer
}

\author{
Junda Wang ${ }^{1,2}$, Lijuan Zhong ${ }^{3}$, Xinjie Zhou ${ }^{1,4}$, Demei Chen ${ }^{1}$, Rui Li ${ }^{1}$ \\ ${ }^{1}$ Department of Ultrasound, The Third Affiliated Hospital, Chongqing Medical University, Chongqing, China; ${ }^{2}$ Department of Radiology, \\ Chongqing Hospital of Traditional Chinese Medicine, Chongqing, China; ${ }^{3}$ Department of Radiology, Leshan People’s Hospital, Leshan, China; \\ ${ }^{4}$ Department of Radiology, The Third Affiliated Hospital, Chongqing Medical University, Chongqing, China \\ Contributions: (I) Conception and design: R Li; (II) Administrative support: R Li; (III) Provision of study materials or patients: J Wang, Y Li; (IV) \\ Collection and assembly of data: J Wang, L Zhong, X Zhou; (V) Data analysis and interpretation: J Wang, D Chen; (VI) Manuscript writing: All \\ authors; (VII) Final approval of manuscript: All authors. \\ Correspondence to: Rui Li, Associate Chief Physician, Professor. Department of Ultrasound, the Third Affiliated Hospital of Chongqing Medical \\ University, Chongqing 401120, China. Email: raylee7991@163.com.
}

Background: Multiphase contrast-enhanced computed tomography (CECT) can reveal the location, morphology, size, and enhancement pattern of gastric cancer (GC), whereas the three-dimensional reconstruction (3DR) technique can better display the relationships of the lesions with surrounding structures, the feeding vessels, and lymph node metastasis. Here, we investigated the value of multi-phase CECT with 3DR in detecting depth of infiltration, lymph node metastasis, and extramural vascular invasion (EMVI) of GC.

Methods: The clinical and imaging data of 132 GC patients admitted to the Chongqing Hospital of Traditional Chinese Medicine and the Third Affiliated Hospital of Chongqing Medical University during the period from January 2012 to October 2019 were collected. All patients received plain and multiphase contrast-enhanced CT scans. The agreement between the results of preoperative CT evaluation and the surgical/pathological findings was compared.

Results: (I) CT findings of GC of 3 differentiation levels: on the multiphase CECT, the peak enhancement percentage was highest in the portal venous phase. The CT values significantly differed among the arterial, portal venous, and equilibrium phases $(\mathrm{P}<0.05)$; the differences in the arterial, portal venous, and equilibrium phases were statistically significant among the well-, moderately, and poorly differentiated groups (all $\mathrm{P}<0.05$ ); finally, the difference in the equilibrium phase was statistically significant between the well- and moderately differentiated groups $(\mathrm{P}<0.05)$. (II) Preoperative CT and postoperative pathology had good consistency in $\mathrm{T}$ staging (Kappa =0.667). (III) The Kappa values between the preoperative CT-diagnosed lymph node metastasis and postoperative pathologically showing an increasing consistency with the increase of CT enhancement differences. (IV) Preoperative CT and postoperative pathology had good consistency in N staging (Kappa =0.779). (V) Preoperative CT in displaying arterial supply to the stomach. The rate of positive EMVI was 32.6\% (43/132) on preoperative CT. The positive EMVI diagnosed by preoperative CT was correlated with tumor size, growth pattern, tissue differentiation degree, $\mathrm{T}$ stage, and $\mathrm{N}$ stage (all $\mathrm{P}<0.05$ ).

Conclusions: Multiphase CECT combined with 3DR has high diagnostic performance in detecting the depth of infiltration, lymph node metastasis, and EMVI of GC.

Keywords: Gastric cancer (GC); infiltration depth; lymph node metastasis; extramural vascular invasion; computed tomography

(C) Journal of Gastrointestinal Oncology. All rights reserved. 
Submitted Apr 02, 2021. Accepted for publication Jun 29, 2021.

doi: 10.21037/jgo-21-276

View this article at: https://dx.doi.org/10.21037/jgo-21-276

\section{Introduction}

Gastric cancer (GC) is one of the most common gastrointestinal malignancies, and most GC patients present with advanced disease. Thus, early diagnosis and accurate preoperative staging are particularly important for GC $(1,2)$. With the evolution of computed tomography (CT) techniques, multiphase contrast-enhanced CT (CECT) and three-dimensional reconstruction (3DT) can be used to evaluate the size, morphology, and infiltration depth of GC lesions; also, these tools can facilitate the preoperative assessment of the blood supply and lymph node metastasis of GC (3-5). Here, we retrospectively investigated the value of multi-phase CECT with 3DR in detecting depth of infiltration, lymph node metastasis, and extramural vascular invasion (EMVI) of GC in 132 GC patients who were treated in our center from January 2012 to October 2019.

We present the study in accordance with the STARD reporting checklist (available at https://dx.doi.org/10.21037/ jgo-21-276).

\section{Methods}

\section{General data}

The clinical and imaging data of 132 GC patients, including 79 males and 53 females aged $41-83$ years, (mean \pm SD: $62.0 \pm 12.5$ years) admitted to the Chongqing Hospital of Traditional Chinese Medicine and the Third Affiliated Hospital of Chongqing Medical University during the period from January 2012 to October 2019, were retrospectively collected. According to the location, these tumors included carcinoma of cardia or fundus of stomach $(n=45)$, gastric body carcinoma $(n=32)$, gastric antrum cancer $(\mathrm{n}=50)$, and diffuse lesions; according to the types, there were 109 cases of gastric adenocarcinoma, 6 cases of adenosquamous carcinoma, 9 cases of signet-ring cell carcinoma, and 8 cases of undifferentiated carcinoma. In terms of metastasis, 76 cases had lymph node metastasis and 56 had no metastasis.

\section{Case inclusion and exclusion criteria}

The inclusion criteria were as follows: (I) presence of gastric lesion(s) on gastroscopy; (II) pathological confirmation of the diagnosis of GC; (III) naive to neoadjuvant radiotherapy or chemotherapy before surgery; (IV) without a previous history of lymph node swelling caused by conditions such as lymph node tuberculosis, lymphoma, or Castleman disease; and (V) having undergone multiphase CECT and with complete thin-layer scan data.

The exclusion criteria included the following: (I) with poor gastric filling, inability to cooperate with the examination, or incomplete imaging information; (II) with iodine allergy or other contraindications; and (III) having received radiotherapy before the operation.

All the subjects signed informed consent for the scan with contrast-enhancement. This retrospective study was approved by the medical ethics committees of the Chongqing Hospital of Traditional Chinese Medicine and the Third Affiliated Hospital of Chongqing Medical University. The study was conducted in accordance with the Declaration of Helsinki (as revised in 2013).

\section{Study methods}

\section{CT examination}

GC patients underwent plain scan and arterial phase, portal venous phase, and equilibrium phase CECT with a GE 64row 128-slice spiral CT scanners (GE Healthcare, USA).

Patients fasted for 6-8 hours. Then, 20 minutes before the examination, they were intramuscularly injected with scopolamine $20 \mathrm{mg}$ and drank $800-1,000 \mathrm{~mL}$ of warm water. Patients were placed in the supine position, and scanned in the area from the diaphragmatic dome to the pubic symphysis. The scanning parameters were as follows: tube voltage $120 \mathrm{kV}$, tube current $250 \mathrm{~mA}$, layer thickness and layer spacing $5 \mathrm{~mm}$, and reconstructed layer thickness $0.625 \mathrm{~mm}$. The contrast agent (iohexol, $350 \mathrm{mgI} / \mathrm{mL}$ ) was injected by inserting a high-pressure syringe (Ulrich Medical, Germany) in the median elbow vein at a flow rate of $3.5-4.0 \mathrm{~mL} / \mathrm{s}$. The dosage was $1.2 \mathrm{~mL} / \mathrm{kg}$, and included $30 \mathrm{ml}$ of saline. Data acquisition was triggered using the bolus tracking technique, with the region of interest being abdominal aorta just below the diaphragmatic dome and the trigger threshold level being set to the CT value of $150 \mathrm{HU}$. The portal venous phase and equilibrium phase 
scanning was performed at 30 seconds and 2.5-3.5 minutes, respectively, after arterial phase scanning.

For image processing, the arterial and portal venous phase images were reconstructed at $0.625-\mathrm{mm}$ intervals and then sent to the workstation (ADW 4.3, GE Healthcare). Coronal and sagittal images were reconstructed with a 3-mm slice thickness. Based on the computer tomography angiography (CTA) results, the gastric and perigastric vessels in the arterial phase were reconstructed using volume rendering (VR) and maximum intensity projection (MIP). MIP was used to reconstruct the peripheral veins of the gastric wall for the portal venous phase images. For data analysis, 2 senior radiologists and 1 gastrointestinal surgeon reviewed the images (including the original axial images and reconstructed images).

\section{Observation indicators and diagnostic criteria}

Tumors were staged according to the American Journal of Cancer Consortium (AJCC) Staging Manual, Eighth Edition (6,7).

\section{T staging of GC by CT}

The location, size, morphology, and infiltration depth of the lesion were observed and measured. The CT value of each phase was measured, and the location and size of the region of interest drawn in each phase were as consistent as possible, with efforts being made to avoid the blood vessels and necrotic areas visible to the naked eye. According to the postoperative pathology, the lesions were divided into a well-differentiated group, moderately differentiated group, and poorly differentiated group.

The criteria for $T$ staging were as follows: $T_{0}=$ no thickening of the and no abnormally enhanced area; $T_{1}=$ tumor invasion of the mucosa or submucosa only of $>5 \mathrm{~mm}$ on the local gastric wall, with even or uneven enhancement in local gastric wall; $T_{2}=$ tumor invasion of the muscular layer and/or subserous layer without involving the serous layer of $>5 \mathrm{~mm}$ on the local gastric wall, with the lesion being slightly, moderately, or obviously enhanced on CECT, and a smooth serous surface layer being present; $\mathrm{T}_{3}=$ tumor invasion of the serous layer without invasion of the surrounding structures of $>5 \mathrm{~mm}$ on the local gastric wall, with the gastric wall having abnormal local morphology, and the lesion being obviously enhanced on CECT; and $\mathrm{T}_{4}=$ tumor invasion of the surrounding tissues and adjacent organs, with disappearance of the serous surface structures, the lesion being poorly demarcated from the surrounding tissues, and organs obviously enhanced on CECT.

\section{$\mathrm{N}$ staging of GC based on lymph node metastasis on CT}

The location, size, maximum and minimum diameters of lymph nodes, and CT values in each phase were measured. The region of interest location and size circled in each phase were maintained as consistently as possible, and the CT enhancement differences in each phase were calculated. According to the literature (8), the CT enhancement difference value was calculated as follows: value = maximum enhancement $\mathrm{CT}$ value on CECT $-\mathrm{CT}$ value on plain scan. Patients were divide into 3 groups: the $\geq 30 \mathrm{Hu}$ group, the $\geq 60 \mathrm{Hu}$ group, and the $\geq 90 \mathrm{Hu}$ group. Metastatic lymph nodes were identified by short diameter $>5 \mathrm{~mm}$ or short-long diameter ratio $\geq 0.7$, moderately or obviously enhanced lymph nodes on CECT, and lowdensity necrotic areas in the central area of lymph nodes on CECT.

The criteria for $\mathrm{N}$ staging were as follows: $\mathrm{N}_{\mathrm{x}}=$ the regional lymph nodes cannot be evaluated; $\mathrm{N}_{1}$ : the cancer has spread to 1-6 lymph nodes; $\mathrm{N}_{2}=$ the cancer has spread to 7-15 lymph nodes; and $N_{3}=$ the cancer has spread to $>15$ lymph nodes.

Tumor invasion to the stomach-feeding arteries and the extramural vessels

The invasion to the stomach-feeding arteries and the extramural vessels was observed; the invasion to gastric arteries was also observed with the application of multiplanar reconstruction (MPR), VR, and MIP techniques in the arterial stage; EMVI was mainly observed on the MPR and MIP images in the portal venous stage and scored by using previously published scoring criteria (9); i.e., a score of $0-2$ indicated EMVI negative and a score of 3-4 indicated EMVI positive.

\section{Statistical analysis}

Data were processed and analyzed in SPSS 21.0 software package (IBM Corp., USA). The normally distributed measurement data are presented using mean \pm SD and nonnormally distributed measurement data as $\mathrm{M} \pm \mathrm{Q}$. The numeration data are presented as numbers and percentages. The agreement between preoperative CT assessment and postoperative pathological findings ( $\mathrm{T}$ stage, $\mathrm{N}$ stage, and metastatic status of GC) was determined by the Kappa 
statistic (generally, Kappa values $<0.4,0.4-0.75$, and $>0.75$ are considered as poor agreement, good agreement, and good agreement, respectively). The comparison of CT values reflecting the differentiation degree of $\mathrm{GC}$ among the 3 groups was determined by analysis of variance (ANOVA), and further intragroup pairwise comparisons were based on the least significant difference (LSD) $t$ test. Correlations between EMVI positivity and relevant clinical indicators were analyzed by using chi-square test. A P value of $<0.05$ was considered significantly different.

\section{Results}

\section{CT findings of GC with 3 different differentiation degrees}

The 132 cases of GC were classified according to the degree of differentiation, with 28 cases $(21.2 \%)$ in the welldifferentiated group, 59 cases $(44.7 \%)$ in the moderately differentiated group, and 45 cases $(34.1 \%)$ in the poorly differentiated group. The lesions were characterized by homogeneously or heterogeneously enhanced single- or multi-layer masses, and their peak enhancement values occurred in the arterial phase in 12 cases (9.1\%), in the portal venous phase in 73 cases $(55.3 \%)$, and in the equilibrium phase in 47 cases $(35.6 \%$ ) (Figures 1,2). In the comparisons among 3 groups of differentiation degree on plain CT or CECT, there was no statistically significant difference in CT values among the 3 groups in the plain phase $(\mathrm{P}>0.05)$; the CT values significantly differed among the arterial, portal venous, and equilibrium phases $(\mathrm{P}<0.05)$; the differences in the arterial, portal venous, and equilibrium phases were statistically significant among the highly, moderately, and poorly differentiated groups (all $\mathrm{P}<0.05$ ); finally, the CT values were not significantly different in the arterial and portal venous phases between the highly and moderately differentiated groups (both $\mathrm{P}>0.05$ ) but significantly differed in the equilibrium phase $(\mathrm{P}<0.05$; Table 1$)$.

\section{Comparison between preoperative CT findings and postoperative pathological $T$ staging results}

Among these 132 GC patients, the overall accuracy of preoperative CT-based T staging was 75.8\% (100/132). More specifically, preoperative CT identified 15 cases of $\mathrm{T}_{1}$, and postoperative pathology confirmed 19 cases of $\mathrm{T}_{1}$, among which 6 cases were overestimated as in $T_{2}$ stage; preoperative CT identified 33 cases of $T_{2}$ and postoperative pathology confirmed 34 cases of $\mathrm{T}_{2}$, among which 8 cases were overestimated as in $\mathrm{T}_{3}$ stage and 2 cases were underestimated as in $\mathrm{T}_{1}$ stage; preoperative $\mathrm{CT}$ identified 45 cases of $T_{3}$ and postoperative pathology confirmed 39 cases of $\mathrm{T}_{3}$, among which 6 cases were overestimated as in $\mathrm{T}_{4}$ stage and 3 cases were underestimated as in $\mathrm{T}_{2}$ stage; and preoperative CT identified 39 cases of $\mathrm{T} 4$ and postoperative pathology confirmed 40 cases of $\mathrm{T}_{4}$, among which 7 cases were underestimate as in $T_{3}$ stage. Consistency between the preoperative CT and postoperative pathological $\mathrm{T}$ staging was average $($ Kappa value $=0.667 ; \mathrm{P}<0.05 ;$ Table 2$)$.

\section{CT findings of the lymph node metastasis of GC}

Based on the CT diagnostic criteria, Preoperative CT identified 1,265 metastatic lymph nodes, while postoperative pathology confirmed 693 metastatic lymph nodes, yielding a diagnostic accuracy of $54.8 \%(693 / 1,265)$. With the CT enhancement difference values set at $\geq 30,60$, and $90 \mathrm{Hu}$, the Kappa values between the preoperative CT-diagnosed lymph node metastasis and postoperative pathologically confirmed lymph node metastasis were $0.285,0.729$, and 0.851 , respectively, showing an increasing consistency with the increase of CT enhancement differences; the consistency between preoperative and postoperative results was good when the difference in CT enhancement was $\geq 90 \mathrm{Hu}$ (Table 3, Figure 1).

\section{Comparison between preoperative CT findings and postoperative pathological $N$ staging results}

Among these 132 GC patients, the overall accuracy of preoperative CT-based N staging was $84.8 \%$ (112/132). More specifically, preoperative CT identified 55 cases of $\mathrm{N}_{0}$ and postoperative pathology confirmed 56 cases of $\mathrm{N}_{0}$, among which 5 cases were overestimated as in $\mathrm{N}_{1}$ stage; preoperative CT identified 43 cases of $\mathrm{N}_{2}$ and postoperative pathology confirmed 42 cases of $\mathrm{N}_{2}$, among which 3 cases were overestimated as in $\mathrm{N}_{3}$ stage and 4 cases were underestimated as in $\mathrm{N}_{0}$ stage; preoperative CT identified 19 cases of $\mathrm{N}_{2}$ and postoperative pathology confirmed 18 cases of $\mathrm{N}_{2}$, among which 2 cases were overestimated as in $\mathrm{N}_{3}$ stage and 3 cases were underestimated as in $\mathrm{N}_{1}$ stage; and preoperative CT identified 15 cases of $\mathrm{N}_{3}$ and postoperative pathology confirmed 16 cases of $\mathrm{N}_{3}$, among which 3 cases were underestimate as in $\mathrm{N}_{2}$ stage. Consistency between the preoperative CT and postoperative pathological $\mathrm{N}$ staging was good (Kappa value $=0.779 ; \mathrm{P}<0.05 ;$ Table 4). 

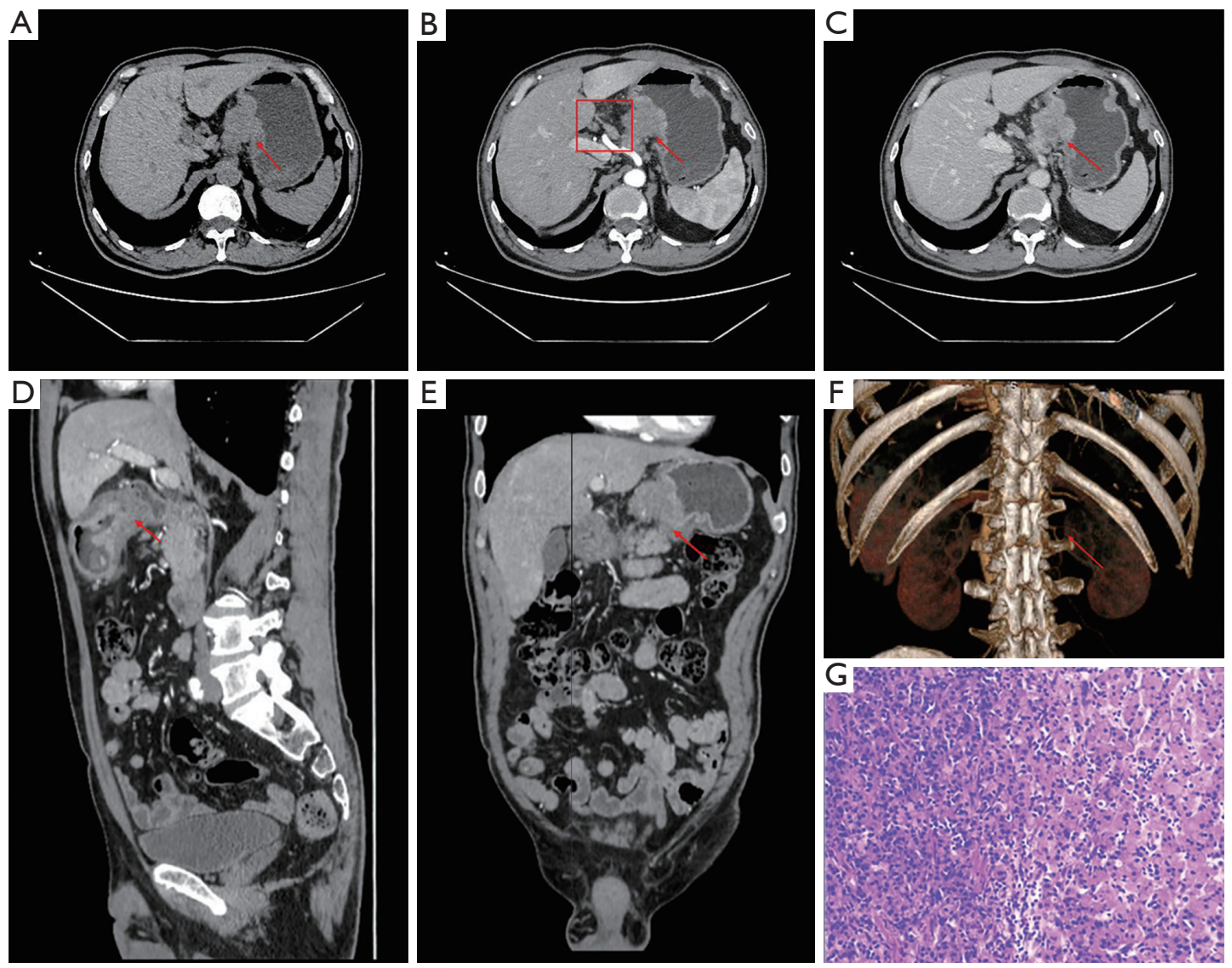

Figure 1 Multistage contrast-enhanced CT scan and 3D reconstruction of poorly differentiated gastric adenocarcinoma. (A) A $4.8 \mathrm{~cm} \times$ $5.2 \mathrm{~cm}$ soft-tissue mass (arrow) seen in the gastric cardia, with a CT value of about $27 \mathrm{Hu}$. (B) Obvious heterogeneous enhancement seen in the arterial phase on CECT, with a CT value of 45-50 Hu. The serous layer is coarse (arrow), and 2 swollen lymph nodes are visible in the hepatogastric space (box). (C) Increased density and heterogeneous enhancement are seen in the equilibrium phase on CECT, with a CT value of 58-62 Hu (arrow), suggesting a CT stage of T4N3Mx. (D) Multiple tiny vessels (arrows) within the tumor are visible on the sagittal plane of $3 \mathrm{D}$ reconstruction, with unclear demarcation between the lesion and adjacent tissues. (E) Tumor invasion of blood vessels is visible on the coronal plane of 3D reconstruction. The involved vessels appear obviously thinner. Extramural vascular invasion (arrow) and 4 swollen lymph nodes in retroperitoneum can also be observed. (F) VR shows unclear demarcation between tumor and blood vessels (arrow). (G) HE $\times 400$, A poorly differentiated adenocarcinoma of the stomach containing signet ring cells and vascular carcinoma thrombus; cancer metastases can be seen in lymph nodes $14 / 15,18 / 18$, and 4/4, and the postoperative stage is T4N3Mx. CT, computed tomography; CECT, contrast-enhanced computed tomography; VR, volume rendering.

\section{Preoperative CT visualization of arterial supply to the stomach}

MPR, VR, and MIP were performed on the arterial phase images before surgery. All of them satisfactorily showed the gastric blood supply, and the visualization rate was $100 \%$ $(132 / 132)$ for the indirect gastric blood supply arteries including the common hepatic artery, proper hepatic artery, splenic artery, and gastroduodenal artery. The visualization rates of gastric direct blood supply arteries were as follows:
$100 \%(132 / 132)$ for the left gastric artery, 96.2\% (127/132) for the right gastric artery, $89.4 \%$ (118/132) for the left gastroepiploic artery, $80.3 \%(106 / 132)$ for the right gastroepiploic artery, $56.8 \%$ (75/132) for the posterior gastric artery, and $52.3 \%$ (69/132) for the short gastric artery.

\section{Preoperative CT visualization of GC EMVI}

Among 132 patients, 43 cases were revealed to be GC 

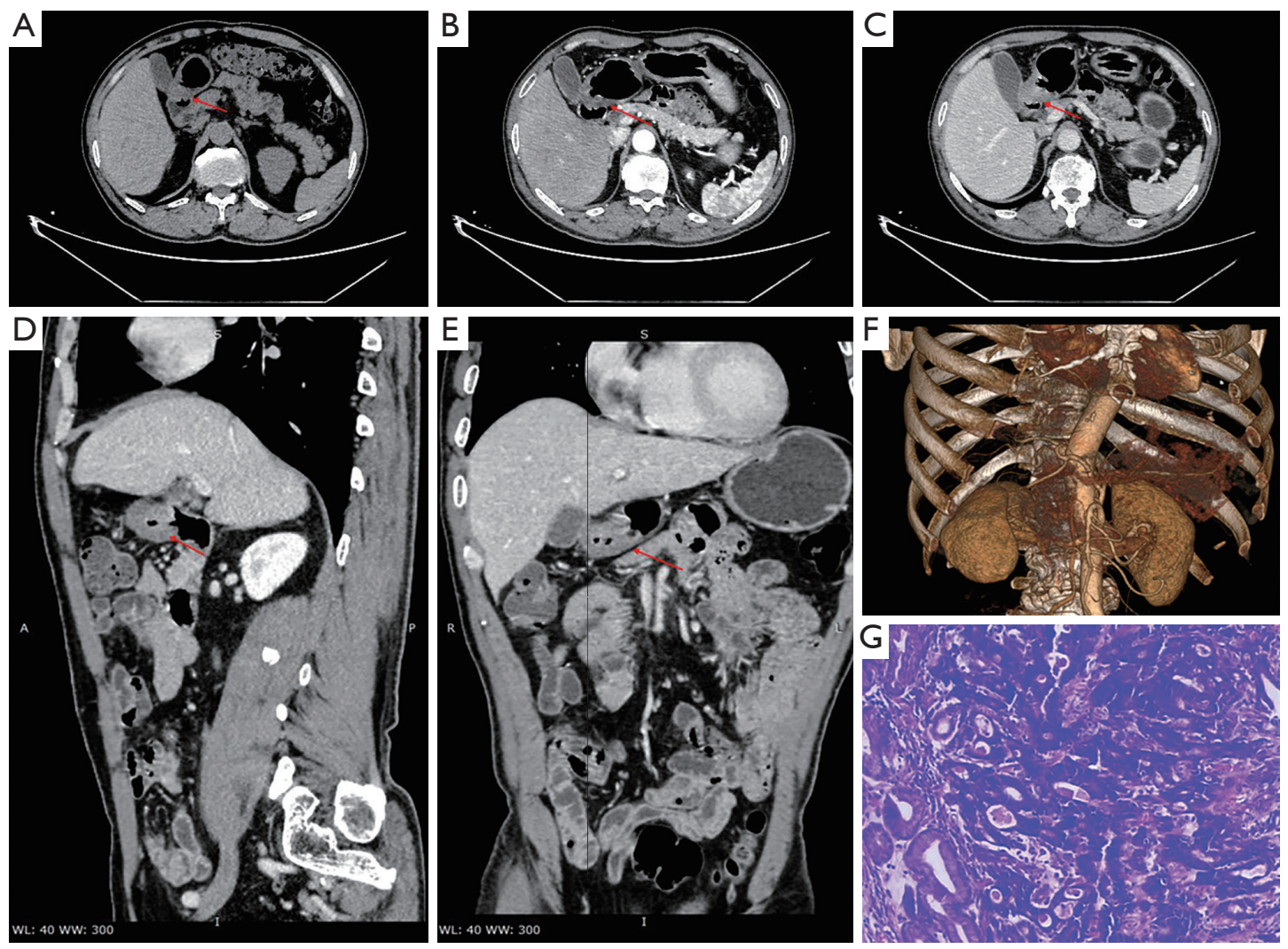

Figure 2 Multi-stage contrast-enhanced CT scan and 3D reconstruction of moderately differentiated gastric adenocarcinoma. (A) A $1.3 \mathrm{~cm} \times 2.1 \mathrm{~cm}$ locally protruding soft-tissue mass (arrow) seen in the gastric antrum, with a CT value of about $26 \mathrm{Hu}$. (B) Mild homogeneous enhancement in the arterial phase was observed following contrast medium administration, with a CT value of about $36 \mathrm{Hu}$ (arrow). (C) Increased density and obvious homogeneous enhancement are seen in the equilibrium phase on CECT, with a CT value of about $50 \mathrm{Hu}$; the serous layer is still clear (arrow), and no obviously swollen lymph nodes can be seen in the neighboring tissues/ organs, suggesting a CT stage of T2N0Mx. (D) No definite tiny vessel shadow can be seen within the tumor on the sagittal plane of 3D reconstruction, and the demarcations (arrows) between the lesion and adjacent tissues are clear. (E) The demarcations (arrows) between lesion and adjacent vessels are clear on the sagittal plane of 3D reconstruction. (F) VR shows clear demarcations (arrows) between tumor and blood vessels. $(\mathrm{G}) \mathrm{HE} \times 400$, For a case of moderately differentiated adenocarcinoma of the stomach, the cancer tissue was located within the mucosa and did not invade the muscular layer; there was no cancer thrombus in the vasculature, pathology did not reveal lymph node metastasis, and the postoperative stage was T2N0Mx. CT, computed tomography; CECT, contrast-enhanced computed tomography; VR, volume rendering.

EMVI positive by preoperative CT, 17 cases with a score of 3 and 26 cases with a score of 4 (Figure 1); and 89 cases were revealed to be negative ( 39 cases with a score of 0,34 cases with a score of 1 , and 16 cases with a score of 2 (Figure 2). Further investigations on the relationships of positive GC EMVI on preoperative CT with the clinical data and $\mathrm{T}$ and $\mathrm{N}$ stages showed that the positive GC EMVI on preoperative CT was not significantly correlated with gender or age (both $\mathrm{P}>0.05$ ), but was correlated with tumor size, growth pattern, and tissue differentiation (all $\mathrm{P}<0.05$; Table 5). Positive GC EMVI status was also significantly correlated with both $\mathrm{T}$ stage and $\mathrm{N}$ stage (both $\mathrm{P}<0.05$ ) (Table 6).

\section{Discussion}

GC is a common malignant tumor, especially in middleaged and elderly populations $(10,11)$. GC progresses 
Table 1 Comparisons of CT values of gastric cancer of different differentiation degrees on plain CT or multiphase CECT

\begin{tabular}{lcccc}
\hline \multirow{2}{*}{ Group } & \multicolumn{3}{c}{ CT value $(\mathrm{Hu})$} \\
\cline { 2 - 5 } & Plain CT & Arterial phase & Portal venous phase & Equilibrium phase \\
\hline Well-differentiated group $(\mathrm{n}=28)$ & $27.05 \pm 1.57$ & $32.64 \pm 1.10$ & $46.37 \pm 3.33$ & $44.89 \pm 2.30$ \\
Moderately differentiated group $(\mathrm{n}=59)$ & $27.14 \pm 1.36$ & $37.06 \pm 2.19$ & $51.35 \pm 4.93$ & $50.12 \pm 3.64^{*}$ \\
Poorly differentiated group $(\mathrm{n}=45)$ & $28.17 \pm 1.53$ & $47.21 \pm 3.88^{\text {*\# }}$ & $62.39 \pm 4.66^{\text {*\# }}$ & 8.733 \\
F value & 7.421 & 18.771 & $0.02 \pm 3.61^{\text {*\# }}$ & 9.183 \\
P value & 0.084 & 0.006 & 0.000 \\
\hline
\end{tabular}

*, $\mathrm{P}<0.05$, compared with well-differentiated group; * $\mathrm{P}<0.05$, compared with the moderately differentiated group. CT, computed tomography; CECT, contrast-enhanced computed tomography.

Table 2 Comparison between preoperative CT findings and postoperative pathological $\mathrm{T}$ staging results $(\mathrm{n}=132)$

\begin{tabular}{lcccccc}
\hline \multirow{2}{*}{ Preoperative CT } & \multicolumn{7}{c}{ Postoperative pathology } & Kappa value \\
\cline { 2 - 5 } & $\mathrm{T}_{1}$ stage & $\mathrm{T}_{2}$ stage & $\mathrm{T}_{3}$ stage & $\mathrm{T}_{4}$ stage & Total & 0.667 \\
\hline $\mathrm{T}_{1}$ stage & 13 & 2 & 0 & 0 & 15 & 0.000 \\
$\mathrm{~T}_{2}$ stage & 6 & 24 & 3 & 0 & 33 & 45 \\
$\mathrm{~T}_{3}$ stage & 0 & 8 & 30 & 7 & 33 & 39 \\
$\mathrm{~T}_{4}$ stage & 0 & 0 & 6 & 40 & 132 \\
Total & 19 & 34 & 39 & & \\
\hline
\end{tabular}

CT, computed tomography.

Table 3 Comparison between preoperative CT enhancement difference and postoperative pathological diagnosis of metastatic lymph nodes

\begin{tabular}{|c|c|c|c|c|c|c|}
\hline $\begin{array}{l}\text { CT enhancement } \\
\text { difference }(\mathrm{Hu})\end{array}$ & \multicolumn{2}{|c|}{ Number of metastatic lymph nodes ( $n$ ) } & Kappa value & $P$ value & Sensitivity (\%) & Specificity (\%) \\
\hline$\geq 30$ & 1,184 & 572 & 0.285 & 0.000 & 68.2 & 21.5 \\
\hline$\geq 60$ & 827 & 598 & 0.729 & 0.000 & 75.3 & 62.7 \\
\hline$\geq 90$ & 259 & 226 & 0.851 & 0.000 & 87.2 & 81.8 \\
\hline
\end{tabular}

CT, computed tomography.

Table 4 Comparison between preoperative CT findings and postoperative pathological $\mathrm{N}$ staging results $(\mathrm{n}=132)$

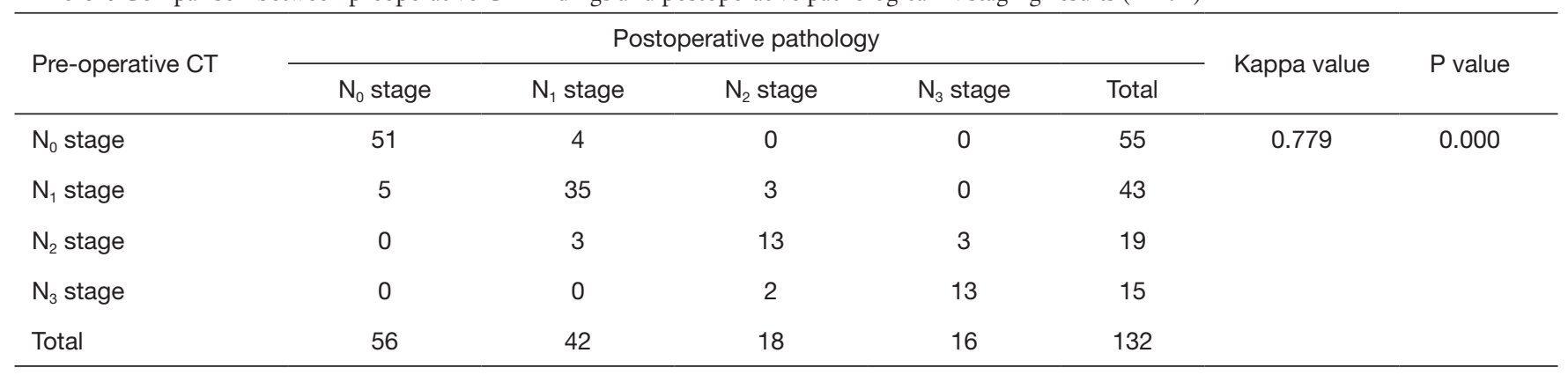

CT, computed tomography. 
Table 5 Relationships of positive GC EMVI on preoperative CT with the clinical data

\begin{tabular}{|c|c|c|c|c|}
\hline Clinical data & $\mathrm{n}$ & Extramural vascular invasion of gastric cancer [n (\%)] & $c^{2}$ value & $P$ value \\
\hline Male & 79 & $27(34.2)$ & & \\
\hline Female & 53 & $16(30.2)$ & & \\
\hline Age (years) & & & 0.045 & 0.831 \\
\hline$>63$ & 75 & $25(33.3)$ & & \\
\hline Tumor size $(\mathrm{cm})$ & & & 9.221 & 0.002 \\
\hline$<5.0$ & 65 & $13(20.0)$ & & \\
\hline$\geq 5.0$ & 67 & $30(44.8)$ & & \\
\hline Proximal nodular + diffuse & 48 & $21(43.8)$ & & \\
\hline Degree of histological differentiation & & & 14.466 & 0.001 \\
\hline Well differentiated & 28 & $4(14.3)$ & & \\
\hline Moderately differentiated & 59 & $15(25.4)$ & & \\
\hline Poorly differentiated & 45 & 24 (53.3) & & \\
\hline
\end{tabular}

GC, gastric cancer; EMVI, extramural vascular invasion; CT, computed tomography.

Table 6 Associations of positive GC EMVI on preoperative CT with T and N stages

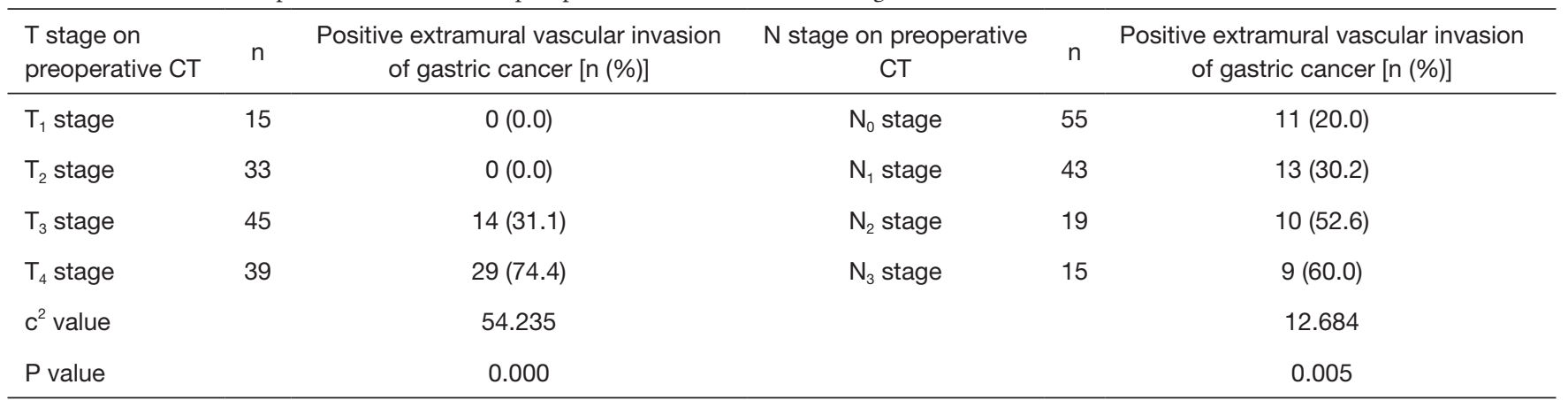

GC, gastric cancer; EMVI, extramural vascular invasion; CT, computed tomography.

rapidly, and thus accurate assessment of its preoperative stage, lymph node metastasis, and EMVI is particularly important for surgical planning and prognostic prediction $(12,13)$. The invasion of cancer into the gastric wall can be clearly demonstrated with high-resolution CT. In addition, multiphase CECT can effectively reveal the lesions and show the lymph node involvements and distant metastases, which enables the accurate staging of GC (14-16).

It is believed that GC enhancement peaks most often in the portal venous phase $(17,18)$. In our current study, comprehensive assessment of the differentiation degree of GC by multiphase CECT improved the accuracy of GC diagnosis; similarly, the CT enhancement differences among GC with 3 different differentiation degrees were the largest in the portal venous phase, and the observation was easiest at the time of peak enhancement. Further pairwise comparisons showed the enhancement degree was significantly higher in the poorly differentiated group 
than in the well- and moderately differentiated groups $(\mathrm{P}<0.05)$, but there was no statistically significant difference between the well-differentiated group and the moderately differentiated group $(\mathrm{P}>0.05)$, which might be explained by the fact that tumor neovascularization was more abundant in the poorly differentiated group than in the well- and moderately differentiated groups. The overall accuracy of preoperative CT-based T staging was 75.8\% (100/132), among which the diagnostic accuracy was the lowest for $T_{1}$ stage, which might have been due to the lesions in $T_{1}$ stage being confined to the mucosal layer or submucosal layer, and the degree of enhancement being low during CECT, making it difficult to judge by the naked eye. Furthermore, poor gastric filling or overfilling during the scans might have also resulted in poor visualization of the layers of the gastric wall, which led to incorrect judgment. The accuracy of preoperative CT diagnosis of $T_{2}$ and $T_{3}$ stages gradually improved, and the possible explanation is that, as the stage increases, the gastric wall at the lesion is obviously thickened, resulting in higher degree of enhancement on CECT and easier diagnosis. The accuracy of preoperative CT diagnosis is the highest for T4 stage due to significant enhancement of the tumor, disappearance of serous layer, and unclear demarcation between lesion and surrounding tissues/organs, which makes the diagnosis much easier. Although the diagnostic agreement was average between preoperative CT and postoperative pathology for $\mathrm{T}$ staging (Kappa value: 0.667), multidirectional, multiangle reconstruction increased the accuracy of $\mathrm{T}$ staging when compared with single-plane observation.

Lymph node metastasis is the most common route of spread for GC (19). While there are still no widely recognized criteria for the diagnosis of lymph node metastasis of GC by CT (20), CT remains an important means to diagnose lymph node metastasis in GC patients. Many studies have explored the agreement of preoperative CT diagnosis and postoperative pathological results, although the results are quite controversial (21-24). Based on the literature (8), metastatic lymph nodes in our current study were identified by short diameter $>5 \mathrm{~mm}$ or shortlong diameter ratio $\geq 0.7$. Among the preoperative CTdiagnosed metastatic lymph nodes, the enhancement difference values were $\geq 30,60$, and $90 \mathrm{Hu}$. The Kappa values between the preoperative CT-diagnosed lymph node metastasis and postoperative pathologically confirmed lymph node metastasis were $0.285,0.729$, and 0.851 , respectively, showing an increasing consistency with the increase of CT enhancement differences; the consistency between preoperative and postoperative results was good when the difference in CT enhancement was $\geq 90 \mathrm{Hu}$. In addition, the overall accuracy of preoperative CT in the $\mathrm{N}$ staging of GC was $84.8 \%(112 / 132)$, and preoperative CT and postoperative pathology had good consistency in $\mathrm{N}$ staging (Kappa $=0.779)$. Several factors might have led to the preoperative overestimation or underestimation of tumor stage. For instance, due to inflammation, the swollen lymph nodes had similar short diameter or enhancement to those of the metastatic lymph nodes, which might have resulted in overestimation; in contrast, some of the swollen lymph nodes were fused into a mass or poorly demarcated from the primary lesion and some of the metastatic lymph nodes might not have met the diagnostic criteria in terms of short diameter, which could have led to underestimation.

Preoperative MPR, VR, and MIP of arterial supply to the stomach can visualize all the indirect gastric blood supply arteries and most of the direct gastric blood supply arteries. Although some of the direct blood supply arteries are too small to be displayed on VR, they can be visualized on MIP images. Thus, the combination of VR and MIP can increase the visualization rate of tiny perigastric vessels. EMVI has been widely believed to be a route for GC metastasis along the neurovascular bundle $(25,26)$. In our current study, the rate of positive EMVI on preoperative CT was $32.6 \%$ (43/132), which was quite consistent with a previous study (21.5\% to $37.0 \%$ ) (27). Moreover, the positive GC EMVI on preoperative CT was not significantly correlated with gender or age (both $\mathrm{P}>0.05$ ) but was correlated with tumor size, growth pattern, and tissue differentiation (all $\mathrm{P}<0.05$ ), which was consistent with the results of other relevant studies $(9,28)$, in which large tumor diameter, distal nodal growth, and poor differentiation were associated with high risk of GC EMVI. In addition, a preoperative CT diagnosis of GC EMVI was significantly correlated with $\mathrm{T}$ and $\mathrm{N}$ stages (especially $\mathrm{T}_{4}$ stage and $\mathrm{N}_{2}$ and $\mathrm{N}_{3}$ stages), which also confirmed that GC spreads along nerves, blood vessels, and lymphatic vessels.

\section{Shortcomings of this study}

This retrospective analysis, (I) in the study of CT preoperative diagnosis of extramural vascular invasion of positive selection, there are higher malignant group than low-grade group, yes, there are significant differences in EMVI; (II) in this retrospective study, the molecular 
characteristics of gastric cancer are missing, so the clinicopathological and molecular characteristics of gastric cancer are missing. The follow-up prospective studies will further explain this part; (III) in this retrospective study, we found that although many patients were diagnosed with gastric cancer, the resources were not complete, such as low, medium and high differentiation, unclear description of intraoperative lymph nodes, unclear invasion of extramural vessels, unclear duration of CT enhancement, and unable to reconstruct the original image. Therefore, only 132 patients were included, resulting in a small amount of data, Next, the team will carry out prospective research; (IV) at present, the relationship between inflammatory factors, immune molecules and clinicopathological characteristics and prognosis of gastric cancer patients has been studied at home and abroad, and the team will further study this direction in the future.

In conclusion, preoperative multiphase CECT combined with 3DR has high diagnostic performance in GC patients for the assessment of the degree of differentiation, $T$ stage, and metastatic lymph nodes and for $\mathrm{N}$ staging. In particular, it has high agreement with postoperative pathology in detecting perigastric vascular invasion and EMVI, with high sensitivity and specificity. Therefore, it is a valuable technique for surgical planning.

\section{Acknowledgments}

Funding: This study received funding from Natural Science Foundation of Chongqing in 2019 (cstc2019jcyjmsxmX0555), Chongqing Science and Health Joint Traditional Chinese Medicine Science and Technology Project in 2018 (ZY201802025), "Xinglin Scholars" Discipline Talent Research Promotion Plan in 2020 (YYZX2020042), Chongqing Traditional Chinese Medicine Hospital second batch of young top-notch talents (CQSZYY2021002).

\section{Footnote}

Reporting Checklist: The authors have completed the STARD reporting checklist. Available at https://dx.doi. org/10.21037/jgo-21-276

Data Sharing Statement: Available at https://dx.doi. org/10.21037/jgo-21-276
Conflicts of Interest: All authors have completed the ICMJE uniform disclosure form (available at https://dx.doi. org/10.21037/jgo-21-276). The authors report that the work was supported by Natural Science Foundation of Chongqing in 2019 (cstc2019jcyj-msxmX0555), Chongqing Science and Health Joint Traditional Chinese Medicine Science and Technology Project in 2018 (ZY201802025), "Xinglin Scholars" Discipline Talent Research Promotion Plan in 2020 (YYZX2020042), Chongqing Traditional Chinese Medicine Hospital second batch of young topnotch talents (CQSZYY2021002). The authors have no other conflicts of interest to declare.

Ethical Statement: The authors are accountable for all aspects of the work in ensuring that questions related to the accuracy or integrity of any part of the work are appropriately investigated and resolved. This retrospective study was approved by the medical ethics committees of the Chongqing Hospital of Traditional Chinese Medicine and the Third Affiliated Hospital of Chongqing Medical University (No. 2019-ky-51). All the subjects signed informed consent for the scan with contrast-enhancement. The study was conducted in accordance with the Declaration of Helsinki (as revised in 2013).

Open Access Statement: This is an Open Access article distributed in accordance with the Creative Commons Attribution-NonCommercial-NoDerivs 4.0 International License (CC BY-NC-ND 4.0), which permits the noncommercial replication and distribution of the article with the strict proviso that no changes or edits are made and the original work is properly cited (including links to both the formal publication through the relevant DOI and the license). See: https://creativecommons.org/licenses/by-nc-nd/4.0/.

\section{References}

1. Ferlay J, Soerjomataram I, Dikshit R, et al. Cancer incidence and mortality worldwide: sources, methods and major patterns in GLOBOCAN 2012. Int $\mathrm{J}$ Cancer 2015;136:E359-86.

2. Yang K, Cho M, Roh CK, et al. Robotic spleenpreserving splenic hilar lymph node dissection during total gastrectomy for gastric cancer. Surg Endosc 2019;33:2357-63.

3. Jiang $M$, Wang $X$, Shan $X$, et al. Value of multi-slice spiral 
computed tomography in the diagnosis of metastatic lymph nodes and N-stage of gastric cancer. J Int Med Res 2019;47:281-92.

4. Zhu ZG. Key points of perioperative whole-process management for patients with advanced gastric cancer. Zhonghua Wei Chang Wai Ke Za Zhi 2020;23:115-22.

5. Wang L, Jin X, Qiao Z, et al. The Value of Low-dose Prospective Dual-energy Computed Tomography with Iodine Mapping in the Diagnosis of Gastric Cancer. Curr Med Imaging 2020;16:433-7.

6. Amin MB, Edge SB, Greene FL, et al. AJCC Cancer Staging Manual[M].8th ed. New York: Springer, 2016:203-20.

7. Amin MB, Greene FL, Edge SB, et al. The Eighth Edition AJCC Cancer Staging Manual: Continuing to build a bridge from a population-based to a more "personalized" approach to cancer staging. CA Cancer J Clin 2017;6:93-9.

8. Joo I, Kim SH, Lee DH, et al. Dynamic ContrastEnhanced Ultrasound of Gastric Cancer: Correlation with Perfusion CT and Histopathology. Korean J Radiol 2019;20:781-90.

9. Yang YT, Dong SY, Zhao J, et al. CT-detected extramural venous invasion is corelated with presence of lymph node metastasis and progression-free survival in gastric cancer. Br J Radiol 2020;93:20200673.

10. Chen Y, Han Y, Du J. Diagnostic challenges in differentiating intramural gastric abscess from gastric cancer: Two case reports. Medicine (Baltimore) 2018;97:e12756.

11. Ma T, Cao LX, Li HJ, et al. Differences of Energy Spectrum CT Findings between Small Cell Lung Cancer with Mediastinal Lymph Node Metastasis and Mediastinal Sarcoidosis. Zhongguo Yi Xue Ke Xue Yuan Xue Bao 2021;43:53-6.

12. Liu S, Shi H, Ji C, et al. Preoperative CT texture analysis of gastric cancer: correlations with postoperative TNM staging. Clin Radiol 2018;73:756.e1-9.

13. Gertsen EC, de Jongh C, Brenkman HJF, et al. The additive value of restaging-CT during neoadjuvant chemotherapy for gastric cancer. Eur J Surg Oncol 2020;46:1247-53.

14. Tsurumaru D, Miyasaka M, Muraki T, et al. Histopathologic diversity of gastric cancers: Relationship between enhancement pattern on dynamic contrastenhanced CT and histological type. Eur J Radiol 2017;97:90-5.
15. Chen X, Yang Z, Yang J, et al. Radiomics analysis of contrast-enhanced CT predicts lymphovascular invasion and disease outcome in gastric cancer: a preliminary study. Cancer Imaging 2020;20:24.

16. Wang Y, Liu W, Yu Y, et al. Potential value of CT radiomics in the distinction of intestinal-type gastric adenocarcinomas. Eur Radiol 2020;30:2934-44.

17. Zhang H, Pan Z, Du L, et al. Advanced gastric cancer and perfusion imaging using a multidetector row computed tomography: correlation with prognostic determinants. Korean J Radiol 2008;9:119-27.

18. Akbas A, Bakir H, Dasiran MF, et al. Significance of Gastric Wall Thickening Detected in Abdominal CT Scan to Predict Gastric Malignancy. J Oncol 2019;2019:8581547.

19. Dong D, Fang MJ, Tang L, et al. Deep learning radiomic nomogram can predict the number of lymph node metastasis in locally advanced gastric cancer: an international multicenter study. Ann Oncol 2020;31:912-20.

20. Chamadol N, Wongwiwatchai J, Bhudhisawasd V, et al. Accuracy of spiral CT in preoperative staging of gastric carcinoma: correlation with surgical and pathological findings. J Med Assoc Thai 2008;91:356-63.

21. Zhang JL, Guo XC, Liu J, et al. Preoperative evaluation using multi-slice spiral CT angiography of right-side colon vascular in laparoscopic radical operation for right colon cancer. Zhonghua Wai Ke Za Zhi 2019;57:927-33.

22. Sun Z, Li J, Wang T, et al. Predicting perigastric lymph node metastasis in gastric cancer with CT perfusion imaging: A prospective analysis. Eur J Radiol 2020;122:108753.

23. Sun Z, Hu S, Li J, et al. An application study of CT perfusion imaging in assessing metastatic involvement of perigastric lymph nodes in patients with $\mathrm{T} 1$ gastric cancer. Br J Radiol 2020;93:20190790.

24. Gao Y, Hu J, Zhang X, et al. Use of Hisense ComputerAssisted Surgery System Enhances Infrapyloric Lymph Node Dissection for Gastric Cancer. J Surg Res 2019;242:31-9.

25. Maehara Y, Kabashima A, Koga T, et al. Vascular invasion and potential for tumor angiogenesis and metastasis in gastric carcinoma. Surgery 2000;128:408-16.

26. Tan CH, Vikram R, Boonsirikamchai P, et al. Extramural venous invasion by gastrointestinal malignancies: CT appearances. Abdom Imaging 2011;36:491-502. 
27. Cheng J, Wu J, Ye Y, et al. Extramural venous invasion detected by MDCT as an adverse imaging feature for predicting synchronous metastases in $\mathrm{T} 4$ gastric cancer. Acta Radiol 2017;58:387-93.

28. Feng C, Cheng J, Xiang H, et al. Value of preoperative abdominal contrast-enhanced multiple-row detector

Cite this article as: Wang J, Zhong L, Zhou X, Chen D, Li R. Value of multiphase contrast-enhanced CT with threedimensional reconstruction in detecting depth of infiltration, lymph node metastasis, and extramural vascular invasion of gastric cancer. J Gastrointest Oncol 2021;12(4):1351-1362. doi: 10.21037/jgo-21-276 computed tomography in predicting the postoperative 1-year disease-free survival for gastric cancer. Zhonghua Wei Chang Wai Ke Za Zhi 2018;21:1059-64.

(English Language Editor: J. Gray) 